\title{
The minimal important difference for residual volume in patients with severe
} emphysema

\author{
Jorine E. Hartman*,\#, Nick H.T. ten Hacken*,\#, Karin Klooster*,\#, \\ H. Marike Boezen ${ }^{\#, \oplus, ~ M a t h i e u ~ H . G . ~ d e ~ G r e e f ~}{ }^{+}$and Dirk-Jan Slebos*,\#
}

ABSTRACT: Residual volume (RV) measured by body plethysmography is a routine measurement in clinical pulmonary practice and is often an important outcome variable in clinical trials. However, it is not known what size of improvement can be regarded as being important in severe emphysema patients. Therefore, the aim of the study is to establish the minimal important difference (MID) for RV in severe emphysema patients undergoing bronchoscopic lung volume reduction.

91 severe emphysema patients were included. RV and total lung capacity (TLC) were measured by body plethysmography. MID estimates were calculated by anchor-based and distribution-based methods. Forced expiratory volume in $1 \mathrm{~s}, 6-\mathrm{min}$ walk distance and St George's Respiratory Questionnaire total score were used as anchors and Cohen's effect size was used as distributionbased method.

The calculated MID estimates according to the different anchors and methods ranged between -0.31 and $-0.43 \mathrm{~L}$ for $\mathrm{RV},-\mathbf{- 6 . 1}$ and $\mathbf{- 8 . 6 \%}$ for percentage change in RV (RV\%) from baseline, and $\mathbf{- 2 . 8}$ and $-4.0 \%$ for RV/TLC.

These MID estimates are useful for sample size determination in new studies on interventions aimed at reducing $\mathrm{RV}$ and for interpreting the results from clinical trials in severe emphysema patients.

KEYWORDS: Chronic obstructive pulmonary disease, hyperinflation, lung volume reduction

ung hyperinflation is an important feature in patients with chronic obstructive pulmonary disease (COPD) and is the result of increased airway resistance, reduced lung recoil and shortened available expiratory time [1]. Lung hyperinflation is strongly associated with important patient-centred outcomes, such as dyspnoea, exercise tolerance and daily physical activity [1,2]. As a consequence, it negatively affects patients' daily functioning and their quality of life.

In the past decade, reducing lung hyperinflation has become an important treatment goal in the management of severe emphysema. Several treatment methods have been shown to improve lung hyperinflation temporarily, such as pursed-lip breathing, exercise training and oxygen use, while bronchodilators or lung volume reduction surgery have been shown to improve lung hyperinflation in patients with COPD more definitively [1, 3]. Lung volume reduction surgery has been shown to improve lung function, quality of life and exercise capacity [4]. Lung volume reduction techniques by bronchoscope are less invasive, and show promising results in improving lung hyperinflation, quality of life and exercise capacity [5-8].

Residual volume (RV) assessed by body plethysmography is commonly used to measure (changes in) lung hyperinflation. Body plethysmography is a routine measurement in clinical pulmonary practice based on Boyle's law, with reproducible measurements of absolute lung volumes. In studies on methods that aim to improve hyperinflation, increased RV constitutes an important inclusion criterion as well as outcome variable. Unfortunately, to date, it is not known what size of improvement can be regarded as being clinically important for the severe emphysema patient.

The concept of minimal important difference (MID) can be used to establish what size of effect in RV measurements after a treatment adequately reflects the perceived improvements by patients. MID can be defined as "a threshold value for a change that would be meaningful and worthwhile by the patients such that he/she would consider repeating the intervention if it were his / her choice to make again" [9]. Any treatment effect above the MID is considered to be relevant.

\section{AFFILIATIONS}

*Dept of Pulmonary Diseases,

\#Groningen Research Institute for Asthma and COPD

'Dept of Epidemiology, University of Groningen, and

${ }^{+}$Dept of Human Movement

Sciences, University of Groningen, University Medical Center Groningen, Groningen, The Netherlands.

\section{CORRESPONDENCE}

D-J. Slebos

Dept of Pulmonary Diseases AA11

University Medical Center Groningen P0 Box 30001

9700 RB Groningen

The Netherlands

E-mail: d.j.slebos@umcg.nl

Received:

Dec 132011

Accepted after revision:

Feb 202012

First published online:

March 222012 
The MID for RV could, therefore, be useful when interpreting the significance of the results of clinical trials for patients, besides the statistical significance of the changes. Additionally, the MID could be used to establish the minimal numbers of subjects to be included in a study to be able to infer meaningful conclusions in future trials (i.e. power calculations). To our knowledge, the MID for RV in patients with severe emphysema has not yet been established.

The aim of this study was to establish the MID for RV in patients with severe emphysema who are being treated by bronchoscopic lung volume reduction.

\section{METHODS}

\section{Study population}

A total of 91 patients with severe emphysema were included in this study. All patients participated in one out of three different bronchoscopic lung volume reduction studies, performed in one hospital in the Netherlands (University Medical Center Groningen, Groningen). Patients were included in this study on MID if they had body plethysmography measurements both from baseline and 1 month after completing the specific bronchoscopic lung volume reduction treatment. 29 patients with severe homogeneous emphysema (10 controls) were included from a randomised, double-blind, sham-controlled trial on bronchoscopic transbronchial airway bypass treatment (www.clinicaltrials.gov identifier number NCT00391612) [10], 33 patients with severe upper- or lower-lobe heterogeneous emphysema (no controls) were included from a bronchoscopic lung volume reduction treatment study using self-expandable nitinol coils (NCT01220908) [6] and 29 patients with severe upper- or lower-lobe heterogeneous emphysema (nine controls) were included from a bronchoscopic lung volume reduction treatment study using one-way endobronchial valves (NCT01101958) [7]. All studies were approved by the local medical ethics committee (University Medical Center Groningen) and all patients gave written informed consent.

\section{Measurements}

RV and total lung capacity (TLC) were measured by body plethysmography (Viasys, San Diego, CA, USA) according to the European Respiratory Society/American Thoracic Society guidelines [11]. Furthermore, all patients performed spirometry (Masterscreen; Viasys) [12], a 6-min walk test [13] and completed the St George's Respiratory Questionnaire (SGRQ) [14]. All measurements were performed at baseline and 1 month after the bronchoscopic lung volume reduction treatment.

\section{MID calculation methods and statistical analysis}

Selected outcome variables for the statistical analyses were RV, percentage change in RV (RV\%) from baseline and the RV/ TLC ratio. Various methods for estimating MIDs have been described in the literature $[15,16]$. Because the combination of multiple methods is generally recommended, our current study included both anchor-based and distribution-based methods to calculate the MID.

Anchor-based methods compare the change in outcome measure with the change in another measure with an established MID ("the anchor") [9]. The anchors chosen in this study were forced expiratory volume in $1 \mathrm{~s}$ (FEV1) (MID $100 \mathrm{~mL}$ [17]), 6-min walk distance (6MWD) (MID $26 \mathrm{~m}$ [18]) and SGRQ total score (MID 4 units [19]). An anchor is suitable to use if there is an appreciable association between the outcome variable and the anchor. Therefore, first, the correlation coefficients between the change in $(\Delta) R V, \Delta R V \%$ and $\Delta R V / T L C$ versus the change in the anchor were assessed. In general, there is no consensus on when a correlation coefficient is considered to be an appreciable association. One review recommends statistically significant $(p<0.05)$ Pearson correlation coefficients of $\geqslant 0.3$ as appreciable [16] and two studies in COPD patients performed the analyses when correlation coefficients were 0.3 or $0.5[18,20]$. Therefore, it is an arbitrary decision, and for the current study, Pearson correlation coefficients of $\geqslant 0.4$ were accepted. Afterwards, linear regression analyses were performed with $\Delta R V, \Delta R V \%$ or $\Delta R V /$ TLC as dependent variables and one of the above-described

\section{TABLE 1 Baseline characteristics and change scores at 1-month follow-up ${ }^{\#}$}

\begin{tabular}{|c|c|c|c|c|c|}
\hline \multirow[t]{2}{*}{ Variable } & \multicolumn{2}{|c|}{ Baseline } & \multicolumn{3}{|c|}{ Change 1 month from baseline ${ }^{\pi}$} \\
\hline & Absolute & $\%$ pred & Absolute & $\%$ change from baseline & $\%>$ MID \\
\hline Females/males & $57 / 34$ & NA & NA & NA & NA \\
\hline Age yrs & $60.1 \pm 8.3$ & NA & NA & NA & NA \\
\hline BMI kg $\cdot \mathrm{m}^{-2}$ & $24.2 \pm 3.3$ & NA & $0.3 \pm 0.8$ & $1.2 \pm 3.3$ & NA \\
\hline FEV $_{1} L^{2}$ & $0.73 \pm 0.3$ & $26.7 \pm 9.8$ & $0.10 \pm 0.17$ & $13.1 \pm 21.2$ & 35.2 \\
\hline 6MWD m & $319.2 \pm 97.5$ & $48.8 \pm 14.7$ & $29.7 \pm 58.6$ & $11.3 \pm 23.2$ & 52.2 \\
\hline SGRQ total score & $62.5 \pm 11.2$ & NA & $-7.8 \pm 12.9$ & $-13.0 \pm 21.5$ & 64.0 \\
\hline RV L & $5.0 \pm 1.1$ & $241.0 \pm 46.6$ & $-0.43 \pm 0.61$ & $-8.7 \pm 12.2$ & Unknown \\
\hline TLC L & $7.8 \pm 1.4$ & $136.5 \pm 14.2$ & $-0.23 \pm 0.43$ & $-2.8 \pm 5.5$ & Unknown \\
\hline RV/TLC \% & $64.3 \pm 8.3$ & $166.8 \pm 23.5$ & $-4.1 \pm 5.7$ & $-6.3 \pm 8.8$ & Unknown \\
\hline
\end{tabular}

Data are presented as $n$ or mean \pm SD, unless otherwise stated. \% pred: \% predicted; MID: minimal important difference; BMI: body mass index; FEV1: forced expiratory volume in 1 s; 6MWD: 6-min walk distance; SGRQ: St George's Respiratory Questionnaire; RV: residual volume; TLC: total lung capacity; NA: not applicable. \#: n=91; $\because$ placebo/control group included. 
anchors as the independent variable. Subsequently, the MID value of the anchor was entered into the equation derived from the linear regression analysis and the MID was calculated from the established equation.

Distribution-based methods compare the change in outcome measure with some measure of variability [9]. In this study, the Cohen's effect size was used. A moderate effect size was calculated of the change score of the outcome measure from baseline to 1 month after the bronchoscopic lung volume treatment.

All statistical analyses were performed using PASW ${ }_{\circledast}$ Statistics 18 (SPSS Inc., Chicago, IL, USA).

\section{RESULTS}

91 patients with severe emphysema, who had undergone body plethysmography measurements at baseline and 1 month later, were included in this study (63\% female; mean age $60 \mathrm{yrs}$ ). Population characteristics at baseline and the 1-month followup changes from baseline are shown in table 1.

\section{MID estimates according to the anchor-based method}

Scatter plots and Pearson correlation coefficients between $\triangle R V$, $\triangle \mathrm{RV} \%$ from baseline and $\triangle \mathrm{RV} / \mathrm{TLC}$ versus $\triangle \mathrm{FEV} 1, \Delta 6 \mathrm{MWD}$ and $\triangle$ SGRQ total score are presented in figure 1. Pearson correlation coefficients ranged from 0.43 to 0.58 . The highest correlations were present with FEV1 (range 0.47-0.58) and the lowest with SGRQ total score (range 0.43-0.47).

The MID estimates derived from the linear regression equations with use of the anchor MID showed that the MID estimates for the absolute RV according to the different anchors were $-0.43 \mathrm{~L}$ (FEV1), -0.41 L (6MWD) and -0.35 L (SGRQ total score) (table 2). The MID estimates for RV\% from baseline according to the different anchors were $-8.6 \%$ (FEV1), $-8.4 \%$ (6MWD) and $-6.9 \%$ (SGRQ total score). The MID estimates for RV/TLC according to the different anchors were $-4.0 \%$ (FEV1), $-3.9 \%$ (6MWD) and $-3.2 \%$ (SGRQ total score).

\section{MID estimates according to the distribution-based method}

The MID estimates calculated with the distribution-based method (Cohen's effect size) were $-0.31 \mathrm{~L}$ for RV, $-6.1 \%$ for $\mathrm{RV} \%$ from baseline and $-2.8 \%$ for RV/TLC (table 2 ).

\section{DISCUSSION}

This is the first study describing MID estimates for lung hyperinflation measurements. Our results indicate MID estimates for RV of $-0.31--0.43 \mathrm{~L}, \mathrm{RV} \%$ from baseline of $-6.1--8.6 \%$ and RV/TLC of $-2.8--4.0 \%$ in patients with severe emphysema
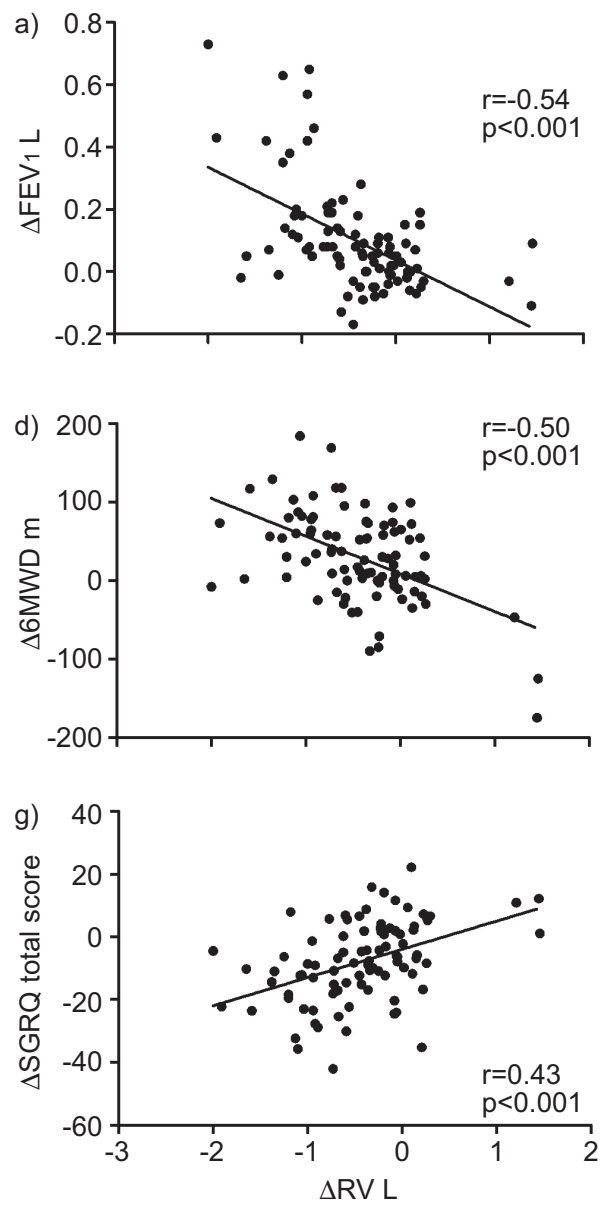

b)

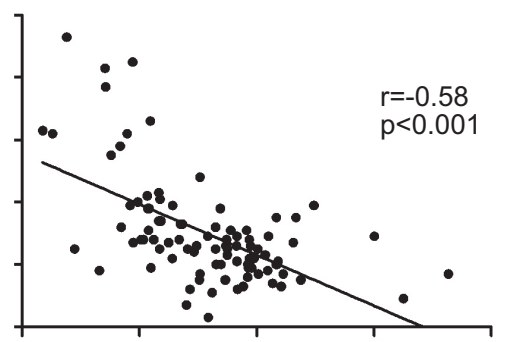

e)

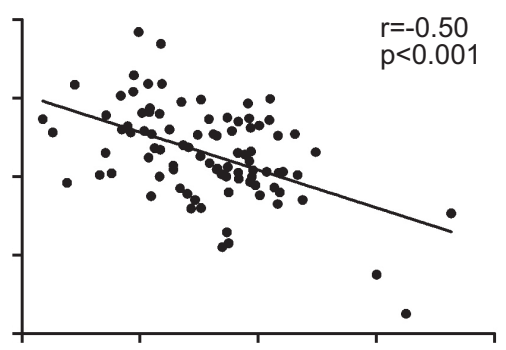

h)

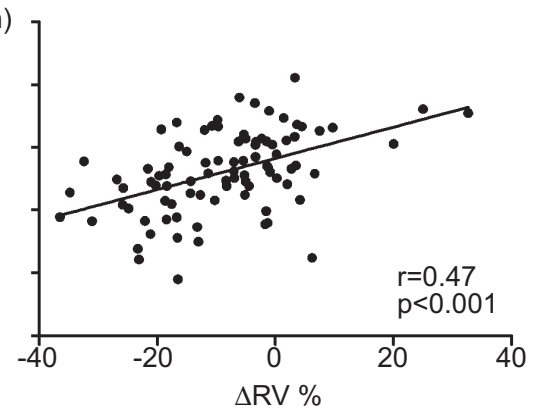

c)

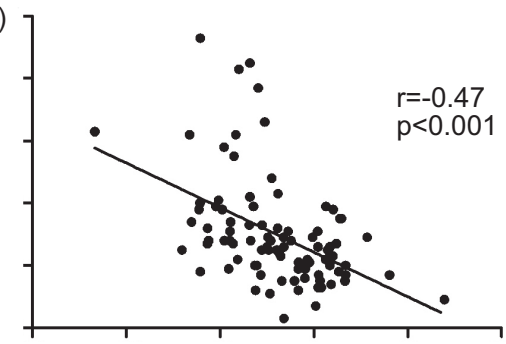

f)

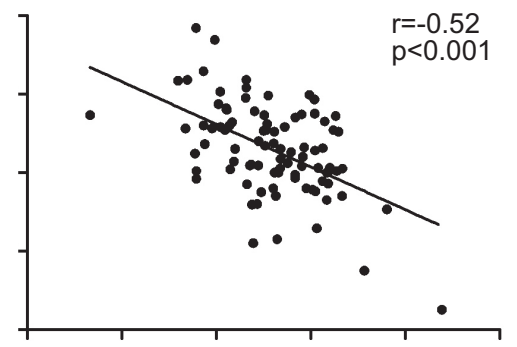

i)

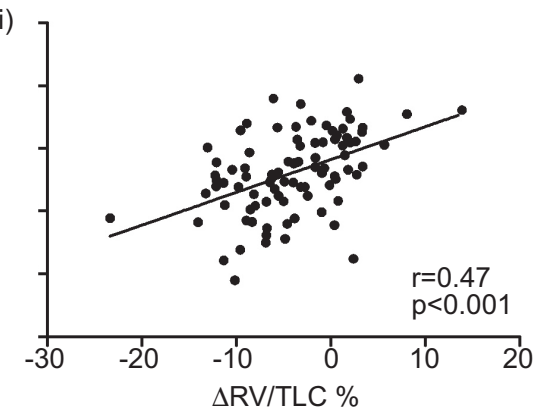

FIGURE 1. Scatter plots of change in lung hyperinflation measurement versus change in anchor variable. $a-c)$ change in ( $\Delta$ ) forced expiratory volume in $1 \mathrm{~s}(F E V 1)$, $d-f)$ $\Delta$ 6-min walk distance (6MWD) and g-i) $\Delta$ St George's Respiratory Questionnaire (SGRQ) total score versus a, d, g) $\Delta$ residual volume (RV), b, e, h) $\Delta$ percentage change in $\mathrm{RV}$ and $\mathrm{c}, \mathrm{f}$ i) $\Delta \mathrm{RV} /$ total lung capacity (TLC). 


\begin{tabular}{|c|c|c|c|}
\hline \multirow[t]{2}{*}{ TABLE 2} & \multicolumn{3}{|c|}{$\begin{array}{l}\text { Minimal important difference (MID) estimates for } \\
\text { residual volume }(\mathrm{RV})\end{array}$} \\
\hline & $\Delta R V L$ & $\Delta R V \%$ & $\Delta R V / T L C \%$ \\
\hline \multicolumn{4}{|l|}{$\begin{array}{l}\text { Anchor-based } \\
\text { method }\end{array}$} \\
\hline $\mathrm{FEV}_{1}$ & $-0.43(-0.62--0.24)$ & $-8.6(-5.7--9.5)$ & $-4.0(-5.9--2.2)$ \\
\hline 6MWD & $-0.41(-0.59--0.23)$ & $-8.4(-4.9--11.9)$ & $-3.9(-5.5--2.3)$ \\
\hline SGRQ total scor & $-0.35(-0.45--0.24)$ & $-6.9(-4.9--8.8)$ & $-3.2(-4.2--2.3)$ \\
\hline $\begin{array}{l}\text { Distribution-base } \\
\text { method }\end{array}$ & -0.31 & -6.1 & -2.8 \\
\hline
\end{tabular}

Data are presented as MID (95\% Cl). $\Delta$ : change; TLC: total lung capacity; FEV1: forced expiratory volume in $1 \mathrm{~s}$; 6MWD: 6-min walk distance; SGRQ: St George's Respiratory Questionnaire.

undergoing bronchoscopic lung volume reduction. MID estimates were determined using both anchor-based and distributionbased methods. High correlations were found between changes in lung hyperinflation measurements and changes in patientcentred outcomes, such as exercise capacity and health-related quality of life, emphasising the importance of this clinical feature for patients with severe emphysema.

Anchor-based and distribution-based methods were used to determine the MID, and for the anchor-based method, three anchors were chosen. Therefore, four MID estimates of each outcome variable were generated. These ranges of MID estimates can be used for power calculations in future trials. Here, we critically evaluate the methods and anchors used for this specific intervention and study population.

For the anchor-based method, it is important that the anchor used is suitable for the analysis. A number of criteria can be used to establish the quality of the anchor. First, the MID of the anchor should be derived from multiple high-quality studies including many well-characterised COPD patients, using multiple methods to establish the MID and agreeing about the final MID estimate. Secondly, the anchor should be derived from a comparable COPD population; thus, for the current study, patients with severe emphysema. Thirdly, the anchor should somehow reflect the perception of improved lung hyperinflation. Finally, the anchor should be highly correlated with changes in RV variables. The anchors used in the current study will be discussed here.

FEV1 seems attractive, as it is a highly reproducible measurement that is strongly associated with RV outcomes. The MID calculation for FEV1 is based on multiple studies, but establishing the MID was never the primary aim of these studies [17]. In addition, the MID of FEV1 was based on a broad variety of COPD patients, including different treatment modalities and, therefore, might not be applicable to our population. Furthermore, the improvement in FEV1 is largely dependent on improvement in vital capacity, which is mainly dependent on a change in $R V$, so $\mathrm{RV}$ and FEV1 are indirectly related. However, the major disadvantage of this anchor is that it does not reflect the perceived improvement by patients [1] and is not a patientreported outcome.
6MWD is highly correlated with RV measurements. Another advantage of this anchor is that the 6MWD MID estimate was calculated from a study investigating the effect of lung volume reduction surgery in severe COPD patients [18] and, thus, is derived from a comparable COPD study population that underwent a comparable, although more invasive, intervention. Furthermore, another study investigated the 6MWD MID using another treatment method and demonstrated a similar MID estimate for pulmonary rehabilitation [20]. Also, the perceived improvement in 6MWD is thought to be a good reflection of the perceived improvement in lung hyperinflation after bronchoscopic lung volume reduction treatment.

SGRQ total score seems more attractive because this instrument is excellently validated for patient-reported outcomes in COPD. However, when using the SGRQ total score as an anchor, the Pearson correlation coefficients were lowest of the three anchors in this study. Another disadvantage is that the MID estimate for SGRQ total score (4 units) is based on multiple studies, using different populations and different MID estimation techniques [19].

A recent study showed that different interventions in different groups of COPD patients may produce different MID estimates [21]. The SGRQ total score from three different lung volume reduction treatment studies were plotted against the change in 6MWD and compared with the effects after pulmonary rehabilitation. A different response pattern for 6MWD and a larger result for health-related quality of life were found between the different treatment modalities. This could indicate that a higher MID estimate for the SGRQ total score for studies using lung volume reduction techniques would be more appropriate. Furthermore, it is important to acknowledge that unblinded interventions can lead to larger improvements in health-related quality of life, due to the fact that patients are aware that they have been treated. Therefore, we advocate that the MID of the SGRQ should subsequently be established in a similar COPD population after a similar treatment, preferably with a placebocontrolled design, before using it as an anchor.

Distribution-based methods do not comply with the primary aim of the MID concept, namely identifying an effect size that is meaningful in the perception of the patient. Therefore, we agree with the consensus that the distribution-based method should only be used to support estimates derived from anchorbased methods $[9,16]$.

In summary, out of the used methods and anchors in the current analyses, the anchor method is the best method to calculate the MID and the 6MWD appears to be the anchor with the highest quality for this specific patient group and intervention.

For the current study, RV\% from baseline and RV/TLC were chosen as outcome variables besides absolute RV. RV\% from baseline was chosen because this adjusts for baseline scores, e.g. it takes sex differences into account. RV/TLC was chosen because it takes individuals' lung capacity into account and has been shown to be an important determinant of improvement in forced vital capacity after lung volume reduction surgery [22]. Unfortunately, most MID estimates are only expressed in absolute numbers. One study that did measure the MID estimate of percentage change from baseline for 
6MWD found a MID estimate of $14 \%$ [20]. However, this MID estimate was based on the effects after a rehabilitation programme and, therefore, was not chosen as anchor in our current study. We recommend that in addition to MID estimates for absolute variables, the percentage change from baseline and percentage predicted should be calculated.

A first limitation of the current study is the relative small sample size. However, all study measurements were performed in one specialised research hospital in the Netherlands, always in the same setting, using the same equipment. This led to highly standardised measurements with low variation, possibly explaining the rather high correlations between changes in lung hyperinflation and the chosen anchors. A second limitation is the measurement of only static lung hyperinflation and not dynamic lung hyperinflation. We anticipate that improved dynamic lung hyperinflation is at least as important for perceived dyspnoea and exercise tolerance as improved static lung volume. Future lung volume reduction studies might, therefore, also investigate correlations between improved dyspnoea scores and improved inspiratory capacities during exercise. A final limitation of the current study is the potentially low generalisability of the MID estimates to other COPD populations or other treatment methods. Our study population is rather homogenous (predominantly female severe emphysema patients) and based on short-term results, which strengthens the MID estimate for this population, but might limit the usefulness for other COPD populations or treatment methods and long-term effects. It is indeed known, at least for SGRQ, that different interventions can lead to different MID estimates in different COPD populations [21]. Therefore, to be able to apply the MID estimates to a more heterogenic COPD population, further research in a larger, more sex-balanced population is needed to investigate whether other interventions that reduce lung hyperinflation, like treatment with bronchodilators, and other COPD populations provide the same MID estimates for RV measurements.

In conclusion, this is the first study that estimated the MID for change in emphysema-related static lung hyperinflation. The calculated MID estimates according to the different anchors and methods ranged between -0.31 and $-0.43 \mathrm{~L}$ for RV, -6.1 and $-8.6 \%$ for $\mathrm{RV} \%$ from baseline, and -2.8 and $-4.0 \%$ for RV/TLC. These MID estimates are useful for sample size determination of new studies on interventions aimed at reducing RV, and for interpreting the results from clinical trials in patients with severe emphysema.

\section{STATEMENT OF INTEREST}

A statement of interest for D-J. Slebos can be found at www.erj. ersjournals.com/site/misc/statements.xhtml

\section{REFERENCES}

1 Cooper CB. The connection between chronic obstructive pulmonary disease symptoms and hyperinflation and its impact on exercise and function. Am J Med 2006; 119: 21-31.
2 Garcia-Rio F, Lores V, Mediano O, et al. Daily physical activity in patients with chronic obstructive pulmonary disease is mainly associated with dynamic hyperinflation. Am J Respir Crit Care Med 2009; 180: 506-512.

3 Puente-Maestu L, Stringer WW. Hyperinflation and its management in COPD. Int J Chron Obstruct Pulmon Dis 2006; 1: 381-400.

4 Tiong LU, Davies R, Gibson PG, et al. Lung volume reduction surgery for diffuse emphysema. Cochrane Database Syst Rev 2006; 4: CD001001.

5 Herth FJ, Gompelmann D, Ernst A, et al. Endoscopic lung volume reduction. Respiration 2010; 79: 5-13.

6 Slebos DJ, Klooster K, Ernst A, et al. Bronchoscopic lung volume reduction coil treatment of patients with severe heterogeneous emphysema. Chest 2011; [Epub ahead of print DOI: 10.1378/ chest.11-0730].

7 Sciurba FC, Ernst A, Herth FJ, et al. A randomized study of endobronchial valves for advanced emphysema. $N$ Engl J Med 2010; 363: 1233-1244.

8 Hopkinson NS, Toma TP, Hansell DM, et al. Effect of bronchoscopic lung volume reduction on dynamic hyperinflation and exercise in emphysema. Am J Respir Crit Care Med 2005; 171: 453-460.

9 Copay AG, Subach BR, Glassman SD, et al. Understanding the minimum clinically important difference: a review of concepts and methods. Spine J 2007; 7: 541-546.

10 Shah PL, Slebos DJ, Cardoso PF, et al. Bronchoscopic lung-volume reduction with Exhale Airway Stents for Emphysema (EASE trial): randomised, sham-controlled, multicentre trial. Lancet 2011; 378: 997-1005.

11 Wanger J, Clausen JL, Coates A, et al. Standardisation of the measurement of lung volumes. Eur Respir J 2005; 26: 511-522.

12 Miller MR, Hankinson J, Brusasco V, et al. Standardisation of spirometry. Eur Respir J 2005; 26: 319-338.

13 ATS Committee on Proficiency Standards for Clinical Pulmonary Function Laboratories. ATS statement: guidelines for the sixminute walk test. Am J Respir Crit Care Med 2002; 166: 111-117.

14 Jones PW, Quirk FH, Baveystock CM. The St George's Respiratory Questionnaire. Respir Med 1991; 85: Suppl. B, 25-31.

15 Guyatt GH, Osoba D, Wu AW, et al. Methods to explain the clinical significance of health status measures. Mayo Clin Proc 2002; 77: 371-383.

16 Revicki D, Hays RD, Cella D, et al. Recommended methods for determining responsiveness and minimally important differences for patient-reported outcomes. J Clin Epidemiol 2008; 61: 102-109.

17 Donohue JF. Minimal clinically important differences in COPD lung function. COPD 2005; 2: 111-124.

18 Puhan MA, Chandra D, Mosenifar Z, et al. The minimal important difference of exercise tests in severe COPD. Eur Respir J 2011; 37: 784-790.

19 Jones PW. St. George's Respiratory Questionnaire: MCID. COPD 2005; 2: 75-79.

20 Holland AE, Hill CJ, Rasekaba T, et al. Updating the minimal important difference for six-minute walk distance in patients with chronic obstructive pulmonary disease. Arch Phys Med Rehabil 2010; 91: 221-225.

21 Troosters T. How important is a minimal difference? Eur Respir 2011; 37: 755-756.

22 Fessler HE, Scharf SM, Permutt S. Improvement in spirometry following lung volume reduction surgery: application of a physiologic model. Am J Respir Crit Care Med 2002; 165: 34-40. 